\title{
Variaciones espaciales y temporales de las comunidades ribereñas de peces en un sistema fluvial no intervenido: Río San Pedro, Cuenca del Río Valdivia (Chile)
}

\section{Spatial and temporal distribution of riparian fish communities in an undisturbed fluvial system: the san pedro river, valdivia basin (Chile)}

\author{
Nicole Colin $^{1 *}$, Priscila PIEdRa $^{1} \&$ EVELYN HABIT ${ }^{1}$ \\ Centro de Ciencias Ambientales, EULA, Universidad de Concepción, Barrio Universitario s/n, Concepción, Casilla 160-C. \\ Chile. \\ *Email: colin.nicole@gmail.com
}

\begin{abstract}
RESUMEN
El conocimiento de la dinámica natural de las comunidades biológicas en sistemas fluviales es relevante para la conservación de estos complejos ecosistemas. El río San Pedro, ubicado en la zona sur de Chile (39 $46^{\prime}-39^{\circ} 51^{\prime}$ S), es un sistema que posee una alta naturalidad dado el bajo nivel de perturbaciones antrópicas significativas. En una condición natural como ésta, el régimen de caudal determina en gran medida la dinámica de sus hábitats en el sentido longitudinal, lateral y temporal del río, influyendo a su vez en la distribución de las especies. De esta forma, la diferenciación hidrogeomorfológica a lo largo del río así como la variación a través del año, genera ensambles altamente dinámicos, tanto a nivel espacial como temporal. Con el fin de conocer la dinámica natural de las comunidades ribereñas de peces, estudiamos la estructura comunitaria a lo largo de tres zonas hidrogeomorfológicas del río San Pedro, denominadas macrozonas. También analizamos la dinámica temporal de los ensambles de peces para conocer cambios estructurales entre las épocas de alto y bajo caudal, asociados a la dinámica de las planicies de inundación. Para la determinación de los patrones espacio-temporales, se realizaron muestreos periódicos en el río San Pedro durante dos ciclos anuales (2006-2007) y se estimaron índices de diversidad clásicos y análisis de agrupamiento basados en similitud. Complementariamente, se realizaron muestreos en diferentes sectores de la cuenca para comparar las tres macrozonas presentes en el río y determinar el grado de singularidad del sistema. Los resultados sugieren la presencia de ensambles ícticos diferenciados a lo largo del río, principalmente entre la macrozona 1 (primer tramo) y 2 (segundo tramo), debido a la predominancia de ambientes más lénticos en la primera y de grandes rápidos en la segunda. Temporalmente, el aumento en las precipitaciones origina ambientes inundados en áreas terrestres. Esto influye en una reducción de la abundancia y diversidad de especies de peces en la época de alto caudal. En contraste, en la época de bajo caudal dominan los ambientes someros, y aumentan tanto el número de individuos como la riqueza específica. La ictiofauna del río San Pedro difiere en su composición y estructura de aquella que habita en otros sectores de la cuenca, lo cual lo convierte en un ecosistema altamente singular.
\end{abstract}

Palabras clave: ecosistema dulceacuícola, estructura de comunidades, macrohábitats, peces, zona ribereña.

\begin{abstract}
The knowledge of the natural dynamic of the biological communities in fluvial systems is relevant for the conservation of these complex ecosystems. The San Pedro River, located in the South of Chile, is a system that has a high naturalness due to the absence of significant anthropogenic intervention. In systems under a natural dynamic like this one, the flow regime determines mostly the habitat dynamics, affecting also the species distribution. This generates highly dynamic assemblages at the spatial and temporal levels. Hence, we studied the fish community structure along three hydrogeomorphological zones of the San Pedro River, named macrozones, to understand the natural dynamic of the freshwater fish communities. In addition, we analyzed the temporal dynamics of the fish assemblages in order to know structural changes between high and low flow seasons, associated to the dynamic of floodplains. Periodic fish samples were done in the San Pedro River during two annual cycles (23 months) to determine the spatial-temporal patterns, and to asses classic diversity indexes and clustering analysis based on similarity. Complementarily, fish samples were collected in different places of the basin to compare the three macrozones occurring in the river and to determine the degree of singularity of the system. The results suggest the occurrence of different fish assemblages along the river, mainly between the macrozone 1 and 2 , due to the predominance of lentic habitats in the first one and large rapids in the second one. Temporarily, the augment in rainfall cause
\end{abstract}


Patrones comunitarios de peces del río San Pedro: Nicole Colin ET AL.

flooded habitats in dry land areas, increasing the extent and heterogeneity of the river habitats. This produces a reduction in the fish species' abundance and diversity during the high flow season. In contrast, shallow environments are dominant during the low flow season, and there is an increment both in the number of individuals and in the specific richness. The San Pedro River's ichtiofauna differs in composition and structure to those in other sectors of the basin, which makes it a highly singular ecosystem.

KEYWORDS: community structure, fish, freshwater ecosystem, macrohabitats, riparian zone.

\section{INTRODUCCIÓN}

Los sistemas fluviales son sistemas complejos, con dimensiones laterales y longitudinales de gran relevancia para la estructuración de las comunidades biológicas (Ward 1989; Junk et al. 1989; Faush et al. 2002). La dimensión lateral se manifiesta con velocidades de corriente máximas en el centro del cauce y velocidades mínimas asociadas a las riberas (Tockner et al. 2010). La dimensión longitudinal, está determinada principalmente por la pendiente, la que a su vez determina la velocidad de corriente, estructurando el sistema lótico (Ward 1989; García de Jalon \& González 1998; Thorp et al. 2006; Lampert \& Sommer 2007). Este gradiente longitudinal se manifiesta también en cambios de las variables fisicoquímicas del agua en el sentido de la corriente, tales como el incremento de la temperatura y concentración de nutrientes o la disminución del oxígeno disuelto (Vannote et al. 1980; Parra et al. 2004). Tanto la dimensión lateral como la longitudinal influyen también en el transporte de sedimentos, determinando así el tipo de sustrato presente en cada tramo del río (Maddock 1999; Newson \& Newson 2000). Este gradiente natural de los ríos genera patrones comunitarios diferenciales de la ictiofauna desde su origen hasta la desembocadura, así como ensambles característicos de la zona ribereña, diferentes a los del centro del cauce (García de Jalón \& González 1998; Habit et al. 2003; Parra et al. 2004; Li \& Gelwick 2005; Lampert \& Sommer 2007; Sullivan \& Watzin 2009). De esta forma, las diferencias existentes en el ambiente físico de un río, fuerzan a las poblaciones de las distintas especies a distribuirse según la existencia de condiciones físicas y bióticas óptimas para su sobrevivencia. Tales condiciones actúan entonces como filtros ambientales, los que determinan la existencia de especies que poseen rasgos funcionales adecuados para cada tipo de ambiente. Ello conlleva finalmente a la formación de un determinado tipo de ensamble (Keddy 1992; Poff 1997), para cada hábitat disponible en el ecosistema.

En este contexto, las zonas ribereñas son hábitats cruciales para la sobrevivencia de las poblaciones ya que constituyen un área de transición entre el ambiente terrestre y acuático, por lo cual es altamente productivo y regula el intercambio de nutrientes entre estos. Como consecuencia de esto, constituye uno de los ecosistemas más importante a nivel mundial, dado además su alta biodiversidad y actividad biogeoquímica (Strayer \& Findlay 2010). Por este motivo el estudio de las comunidades ribereñas constituye un factor esencial para el conocimiento de la dinámica biótica de estos sistemas.

En Chile, los ríos andinos se caracterizan por ser de corta extensión y presentar un perfil longitudinal marcadamente cóncavo, con ambientes ritrales y potamales presentes en cortas distancias. Entre los ríos de origen andino en Chile, el río San Pedro, tributario de la cuenca del Valdivia (39 $46^{\circ}$ - 39 $51^{\prime}$ 'S), no cumple exactamente con tal patrón general, ya que nace de una larga cadena de ocho lagos (Niemeyer \& Cereceda 1984). En consecuencia, el primer tramo del río no presenta características de un río ritral de bajo orden, sino atributos transicionales desde lacustres a fluviales. De esta forma, se reconocen tres macrohábitats o macrozonas hidrogeomorfológicas a lo largo del río. Una zona alta, de influencia lacustre; una zona media ritral y una zona baja hiporitrónica o de transición. Por otra parte, este sistema se caracteriza por presentar una mínima intervención antrópica a lo largo de todo su cauce, y un régimen de caudal natural, sin regulación, más que la lacustre. Esta característica de alta naturalidad, casi única en la depresión intermedia del centro sur de Chile, convierte al río San Pedro en un sistema ideal para el estudio de patrones comunitarios de peces en condiciones naturales.

La ictiofauna chilena es altamente singular, caracterizándose por ser poco diversa, poseer tamaños corporales pequeños, no distribuirse sobre los $1500 \mathrm{~m}$, y tener un alto nivel de endemismo (Campos 1993; Dyer 2000; Vila et al. 2006). De igual manera, esta fauna se caracteriza porque un gran porcentaje presenta problemas de conservación, principalmente debido a la pérdida de hábitat (Habit et al. 2006; 2007). Por ello, el conocimiento de la estructura y dinámica de los ensambles naturales de peces es fundamental para una posterior aplicación de medidas de mitigación y restauración de hábitats, frente a eventuales alteraciones antropogénicas.

Considerando estos antecedentes, analizamos la relación de la zonación física del río San Pedro con la estructura espacio-temporal de su fauna de peces. Dada entonces 
la diferenciación hidrogeomorfológica a lo largo del río San Pedro así como la variación temporal del caudal y la influencia de esta dinámica en la ictiofauna, estudiamos la estructura de los ensambles de peces en los tres macrohábitats (o macrozonas) durantes dos ciclos anuales en diferentes sitios a lo largo del río. Complementariamente, analizamos la estructura comunitaria de la ictiofauna a lo largo de la cuenca hidrográfica del río Valdivia, para así determinar el grado de singularidad del ensamble de peces del río San Pedro.

\section{MATERIALES Y MÉTODO}

ÁrEA DE ESTUDIO

El estudio se realizó en el río San Pedro, desde su origen, en la desembocadura del Lago Riñihue hasta aproximadamente $200 \mathrm{~m}$ aguas abajo de la zona de confluencia del río San Pedro con el río Quinchilca (3946' - 3951' S) (Fig. 1). En total, el área de estudio abarcó una extensión de $40 \mathrm{~km}$. Para determinar la distribución longitudinal de la fauna íctica se caracterizó el ensamble de peces en cada uno de los tres macrohábitats presentes, definidos como macrozonas, de acuerdo a su pendiente (Fig. 2). Para ello se estimó el promedio de tres mediciones del seno del ángulo expresado en porcentaje, el cual es definido como la proporción del cambio de altitud a la longitud de la superficie entre dos puntos, en cada punto de muestreo. La macrozona 1 (M1), se encuentra en el primer tramo del río y se caracteriza por presentar influencia lacustre, de flujo sin rápidos evidentes. La macrozona 2 (M2) constituye la parte encajonada, con mayor pendiente y velocidad de corriente, constituyendo áreas de secuencias de rápidos profundos y pozas. Por último, la macrozona 3 (M3) abarca el último tramo del río, es un área mixta de rápidos y áreas de menor velocidad. Los muestreos se realizaron durante dos ciclos anuales para determinar patrones temporales, de la fuerte dinámica interestacional en sistemas fluviales andinos. En total se realizaron 465 eventos de pesca, durante 289 días (octubre de 2005 hasta marzo de 2008). Para la toma de datos se establecieron 22 sitios de muestreo, los cuales fueron distribuidos de acuerdo a las condiciones de accesibilidad y representatividad de cada macrozona (Tabla 1). Los muestreos fueron realizados solo en la zona ribereña, considerándose como tal, los ambientes de hasta $1 \mathrm{~m}$ de profundidad con velocidades de corriente $<1 \mathrm{~m} / \mathrm{s}$. El arte de pesca utilizado para la captura de los individuos fue pesca eléctrica (LR-24 Electrofisher, Smith Root, Inc), con redes bloqueadoras, entre 100-150 $\mathrm{m}$. El tiempo de pesca fue variable entre un sitio u otro. Una vez capturados los individuos fueron puestos en estanques con agua, con un recambio constante de ésta y un suministro adicional de oxígeno para mantener una temperatura y oxigenación similar a la del río al momento de ser capturados. Posteriormente fueron determinados taxonómicamente hasta nivel de especie y contabilizados in situ. Finalmente, todos los individuos fueron devueltos a su hábitat original de captura.

Tabla 1. Puntos de muestreo de cada macrozona en el río San Pedro y sus respectivas coordenadas (UTM).

Table 1. Sample sites trough San Pedro River, indicating the macrozone which belong and their geographical coordinates (UTM).

\begin{tabular}{cllcc}
\hline \multirow{2}{*}{ Macrozona Nombre del Sitio } & Código & UTM & UTM \\
& \multirow{2}{*}{$\mathbf{1}$} & X & Y \\
& Isla Grande & IG & 718328 & 5594046 \\
& Hotel & HO & 717859 & 5594106 \\
& Desembocadura Catalina & DC & 717642 & 5594034 \\
& Piedra Blanca & PB & 717526 & 5594451 \\
\hline $\mathbf{2}$ & Los Bajos & LB & 716917 & 5595892 \\
& Carmen del Trafún Este & TE & 715426 & 5596941 \\
& Carmen del Trafún Oeste & TO & 712171 & 5597029 \\
& Champulli & CH & 708350 & 5596457 \\
& Forestal Maitén & FM & 707795 & 5596191 \\
& El Maitén & MA & 707240 & 5596014 \\
& La Quinta & LQ & 706945 & 559575 \\
& Nuevo Maitén & NM & 706548 & 5596095 \\
& Cun-Cun Oeste & CO & 703088 & 5597452 \\
& Anita María & AM & 697222 & 5595025 \\
& Chacaipulli & CP & 695988 & 5593261 \\
& Balsa Norte & BN & 695694 & 5592383 \\
\hline Balsa Sur & BS & 694813 & 5591543 \\
El Mosqueto & EM & 694546 & 5590769 \\
& Cuyincahuin & CU & 693429 & 5586939 \\
& Camping & CA & 692278 & 5586120 \\
& San Pedro & SP & 691787 & 5586085 \\
Quinchilca & QI & 692029 & 5586006 \\
\hline
\end{tabular}


Con el objetivo de conocer la singularidad de los ensambles del río San Pedro con respecto al resto de la cuenca hidrográfica del río Valdivia, se realizaron muestreos en 50 sitios a lo largo de la cuenca (Fig. 1). Estos correspondieron a 27 sitios ubicados en ríos aguas arriba (Arrb) del río San Pedro, 9 en tributarios del río San Pedro (Tr) y 14 aguas abajo del río San Pedro, abarcando tanto el río Calle Calle (Aab) como sus tributarios ( $\operatorname{TrAab})$.

\section{ANÁLISIS DE DATOS}

Los datos de abundancia bruta fueron transformados a captura por unidad de esfuerzo (CPUE), estimada como número de individuos * 100 / tiempo de pesca en horas * área muestreada en $\mathrm{m}^{2}$. De esta manera se obtuvo un índice de abundancia comparativa para todos los sitios y fechas muestreadas. Se analizó la estructura de los ensambles tanto espacial como temporalmente estimando la riqueza específica, diversidad (índice de Shannon) y equidad (índice de Pielou) para cada sitio, mediante el software Primer-E v6.15 (Clarke \& Gorley 2006). Para determinar si existían diferencias significativas en estos índices entre macrozonas del río y épocas de alto y bajo caudal, se utilizaron análisis de varianza multivariada (MANOVA) en el programa STATISTICA, previa verificación de la normalidad de los datos. Las épocas de alto y bajo caudal se definieron sobre la base de la información hidrológica mensual (octubre 2005 a marzo 2008) de la estación fluviométrica del río San Pedro en desagüe del lago Riñihue de la DGA. Sobre la base de esa información se agruparon los meses de diciembre a abril como época de bajo caudal y de mayo a noviembre, como época de alto caudal. Para analizar si existían diferencias significativas en la estructura comunitaria de peces entre las distintas macrozonas y las épocas de alto y bajo caudal, se utilizaron análisis de similitud (ANOSIM) basados en la matriz de disimilitud de Bray-Curtis, sobre la abundancia (CPUE) estandarizada y transformada a raíz cuadrada para reducir el peso de las especies dominantes. Para determinar qué especies son las que contribuyen a explicar las agrupaciones y la disimilitud entre macrozonas y épocas, se utilizó un análisis de SIMPER (Similarity Percentages). Por último, para analizar la similitud de las comunidades de peces del río San Pedro con aquellas presentes en otras zonas de la cuenca hidrográfica, se utilizó un análisis de escalamiento multidimensional no métrico basado en la matriz de disimilitud de Bray Curtis. Dadas las diferencias en el esfuerzo de muestreo entre los sitios del río y la cuenca, el análisis se realizó sobre los datos de presenciaausencia, para hacerlos comparables, utilizando el software Primer-E v6.15 (Clarke \& Gorley 2006).

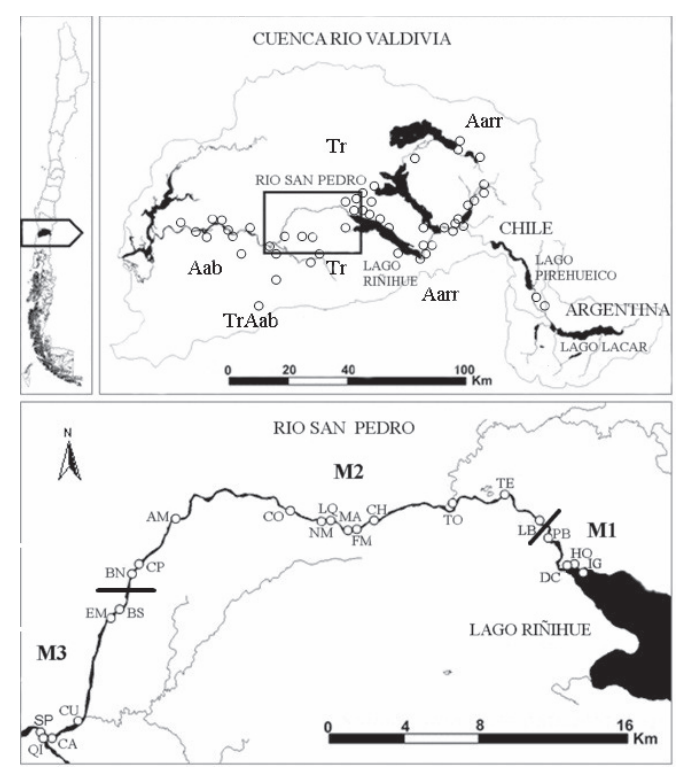

Figura 1. Mapas del área de estudio. Arriba, Cuenca del río Valdivia. El recuadro indica la extensión del río San Pedro y los círculos los sitios de muestreo fuera del río San Pedro (Aarr: aguas arriba, Aab: aguas abajo, Tr: tributarios, Trab: tributarios aguas abajo). Abajo se muestra el río San Pedro desde el desagüe del Lago Riñihue hasta la confluencia con río Quinchilca y los 22 sitios de muestreos señalados con un círculo blanco y su respectiva simbología (ver Tabla 1). Las líneas negras indican los límites de las macrozonas. M1: macrozona 1, M2: macrozona 2 y M3: macrozona 3.

Figure 1. Map of the study area. Overhead picture shows the Valdivia River basin showing sampling sites up and downstream from the San Pedro River (Aarr: basin upstream , Aab: basin dowstream, Tr: tributary streams, Trab: trybutary streams below San Pedro River). Below, the San Pedro River from its mouth in the Riñihue Lake outlet to the confluence with the Quinchilca River, and the 22 sample sites indicated with a white circle and site symbol (see Table 1). Dark lines indicate the macro-zone deadlines. M1: macrozone 1, M2: macrozone 2 y M3: macrozone 3. 


\section{RESULTADOS}

Se registraron 16 especies de peces en el río San Pedro, de las cuales 14 son nativas y dos son introducidas (Fig. 3). De éstas, Aplochiton zebra Jennys, 1842 sólo fue detectada en el área de estudio a partir de octubre del 2006, es decir, un año después de iniciados los muestreos, lo cual sugiere su baja abundancia en el área. Por otra parte, Hatcheria macraei Girard, 1855 si bien es una especie que fue detectada en el área, para efecto de los análisis no fue considerada debido a que no existe certeza de su abundancia. Se capturó un total de 36.756 individuos, de los cuales el $95 \%$ correspondía a alguna de las especies nativas y sólo un 5\% a ejemplares de las especies introducidas. Las especies de mayor abundancia fueron Percilia gillisi Girard, 1854 y Galaxias maculatus Jennys, 1842, las que representaron el $38 \%$ del total.

La distribución espacial de los ensambles de peces fue consistente con las tres macrozonas hidrogeomorfológicas identificadas (ANOSIM, Rglobal $=0,12, \mathrm{p}=0,001$ ), encontrándose una mayor diferencia entre las comunidades de peces de la macrozona 1 y 2 (ANOSIM, $\mathrm{R}_{1-2}=0,18$ $, \mathrm{p}=0,001 ; \mathrm{R}_{1-3}=0,072, \mathrm{p}=0,001 ; \mathrm{R}_{2-3}=0,078, \mathrm{p}=$ $0,002)$. Las especies que mejor caracterizan el ensamble de la macrozona 1, correspondieron a G. maculatus, Galaxias platei Steindachner, 1898 y Cheirodon australe Eigenmann, 1927 (Tabla 2). La macrozona 2, en tanto, queda caracterizada por Diplomystes camposensis Arratia, 1987 y Trichomycterus areolatus Valenciennes, 1840. G. maculatus también mostró un alto porcentaje de contribución para esta macrozona debido a su alta abundancia en pequeños remansos entre los grandes rápidos (Tabla 1). La ictiofauna que caracteriza la macrozona 3, estuvo representada por $G$. maculatus, T. areolatus, G. platei y Geotria australis Gray,
1851. Por último, dada su alta abundancia a lo largo del río P. gillissi caracterizó las tres macrozonas (Tabla 2).

Los índices de diversidad presentaron una diferencia significativa global a lo largo del río (MANOVA Wilks Lambda $\left._{(8,2,56)}=0,953, \mathrm{p}=0,0009\right)$, (Fig. 5). Los análisis $a$ posteriori mostraron que estas diferencias son significativas para los índices de diversidad (ANOVA, $\mathrm{F}=4,39, \mathrm{p}$ $=0,012$ ) y equidad de especies (ANOVA, $\mathrm{F}=5,15, \mathrm{p}=$ 0,006 ), entre las macrozonas 1 y 2 (Tukey, $p=0,004, p=$ $0,003)$ con valores significativamente menores en el primer macrohábitat (Fig. 4).

El patrón temporal de la estructura comunitaria muestra una clara tendencia cíclica, con una dinámica temporal anual marcada (Fig. 5). Existe una mayor riqueza y diversidad en la época estival, principalmente en el mes de febrero, lo cual coincide con la época de bajo caudal. En tanto, en la época de alto caudal estos parámetros disminuyen notablemente (MANOVA Wilks Lambda $_{(4,2,95)}=0,973, \mathrm{p}=0,02$ ). La comunidad de peces presente en la época de alto caudal esta caracterizada principalmente por especies que se encuentran presentes en hábitat de pozas (Tabla 2) y es significativamente diferente a la de la época de bajo caudal (ANOSIM, Rglobal = $0,1, p=0,001)$. De mayor a menor porcentaje de contribución, las especies que componen la comunidad de alto caudal son G. maculatus, G. platei y P. gillissi. Por otra parte los peces más abundantes en la época de bajo caudal, se caracterizan por ocupar el canal del río. Las especies que caracterizan a este ensamble son P. gillissi, T. areolatus, G. maculatus y D. camposensis. Si bien $P$. gillissi también ocupa el ambiente terrestre inundado presente solo en esta época, hace un uso más extensivo del lecho de río. Con G. maculatus ocurre lo contrario, ocupa más intensivamente las planicies inundadas y en menos medida el lecho de río (Tabla 3).

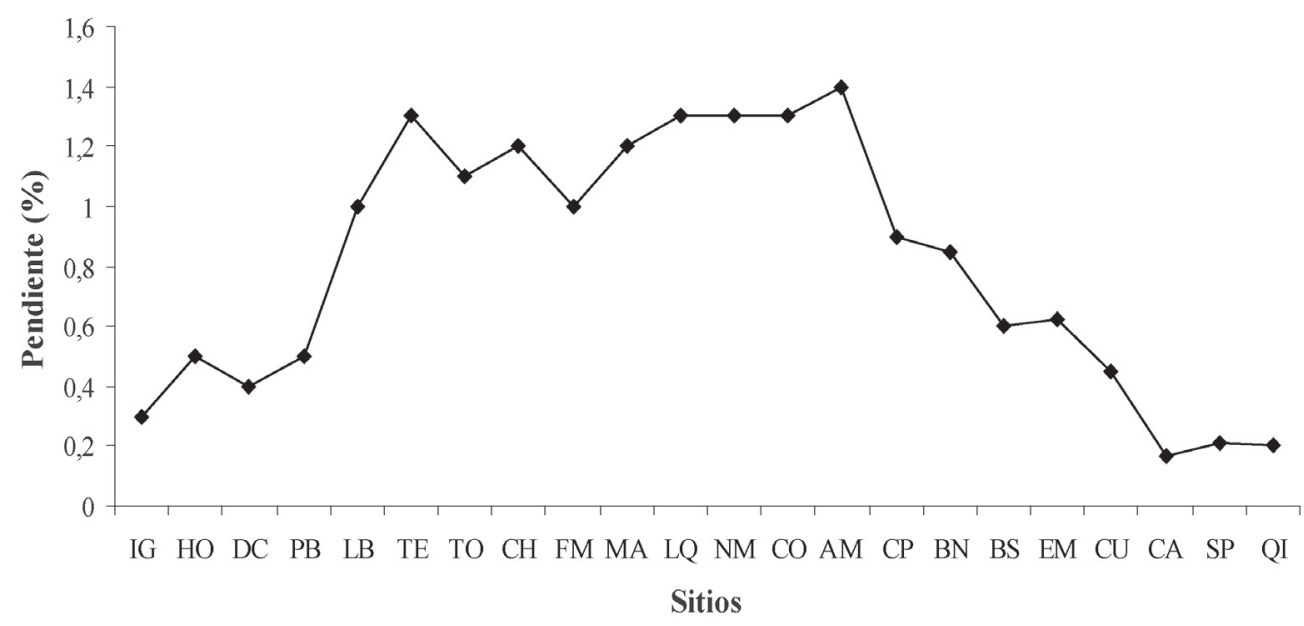

Figura 2. Valores de pendiente del río en cada sitio de muestreo. Las líneas indican el límite de las macrozonas.

Figure 2. Slope values of the river in each sample site. Lines indicate the deadline of macrozone. 


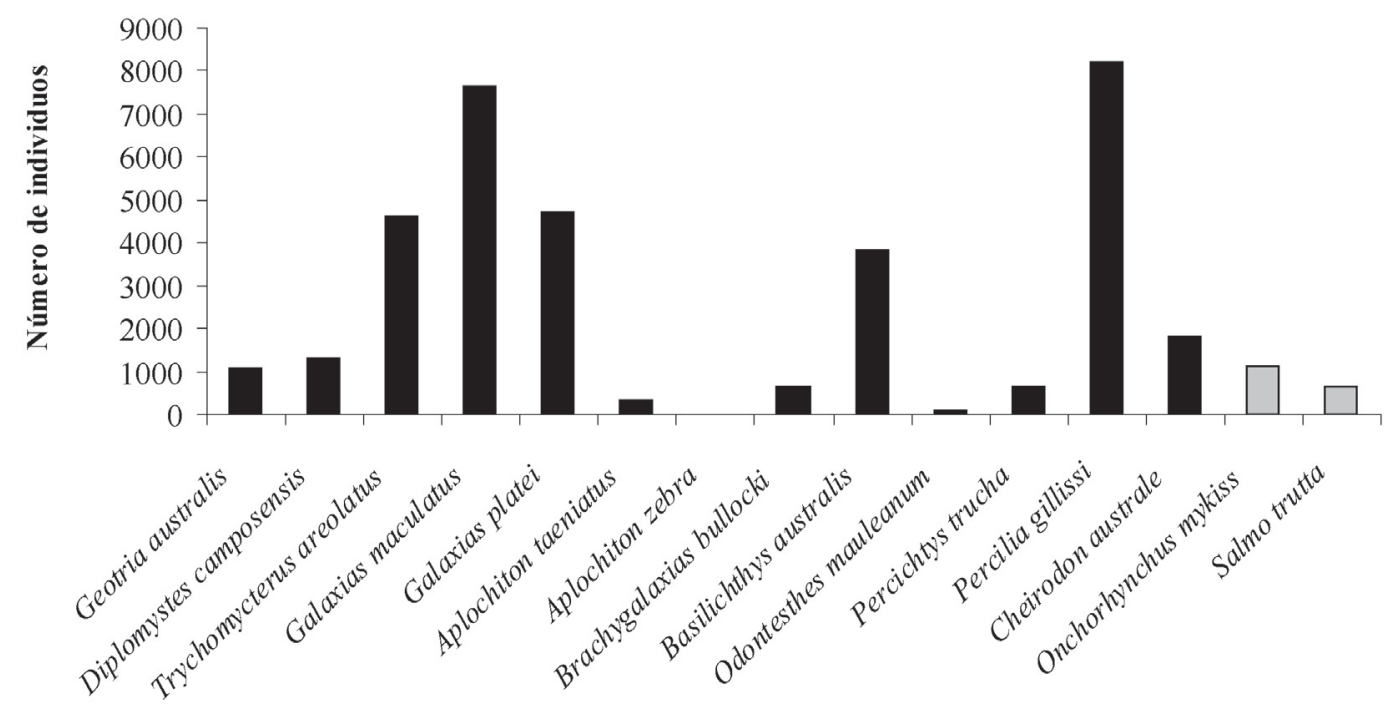

Figura 3. Número de individuos capturados (y devueltos) entre octubre de 2005 y marzo de 2008 en el río San Pedro. En negro se indican las especies nativas y en gris las introducidas.

Figure 3. Number of individual captured from October 2005 to March 2008 in the San Pedro River. Native species in black and introduced species in grey.

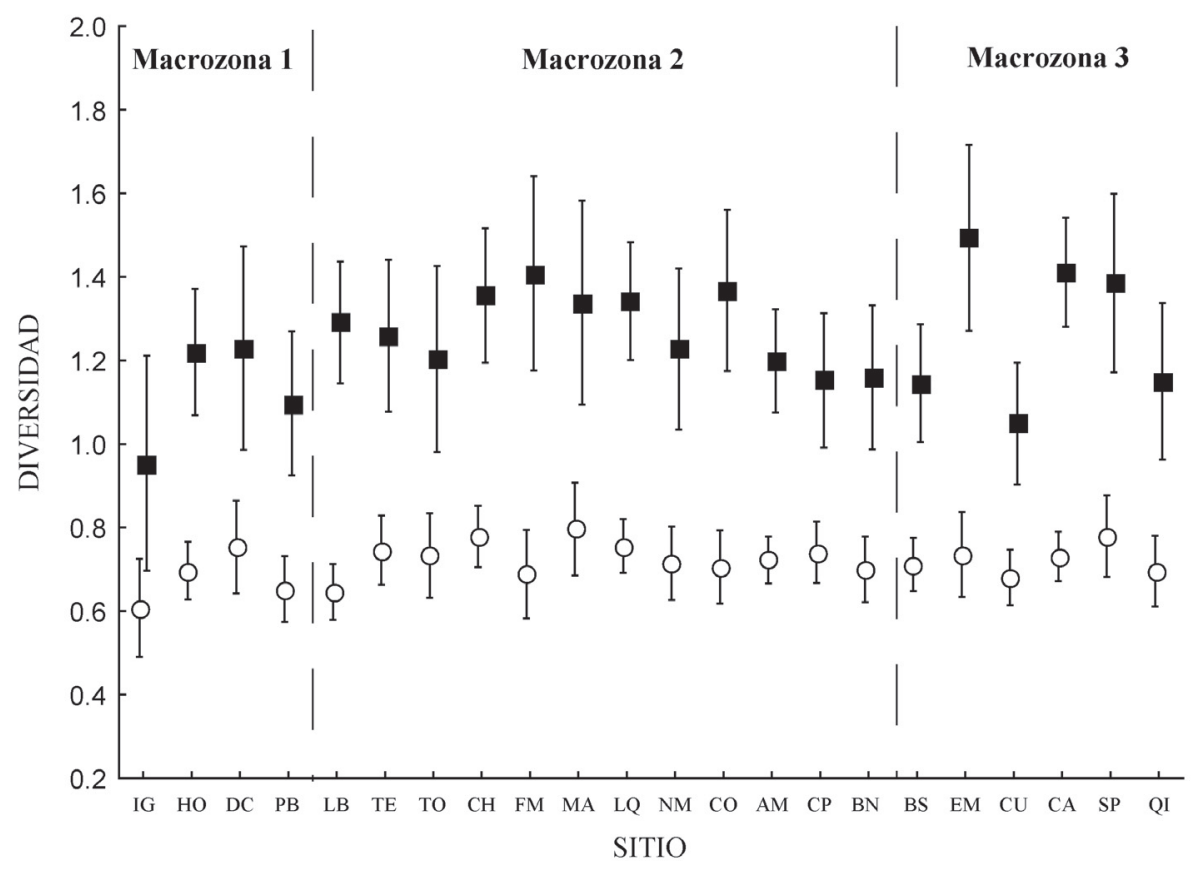

Figura 4. Índices de Equidad (○) y de diversidad de Shannon (a), (promedio \pm error estándar), a través de los sitios de muestreo desde el desagüe del lago Riñihue hacia aguas abajo hasta la confluencia con el río Quinchilca. Las líneas punteadas indican el límite de las macrozonas. Las siglas corresponden a los sitios de muestreo (ver Figura 1) (N=31344).

Figure 4. Values of evenness ( $\circ$ ) and Shannon diversity ( $\bullet$ ) (mean \pm standard error), of each sample sites from the Riñihue lake oulet and downstream until the confluence with the Quinchilca River. Dotted line shows the macrozones limits. X axis shows sample sites code (see Figure 1). 


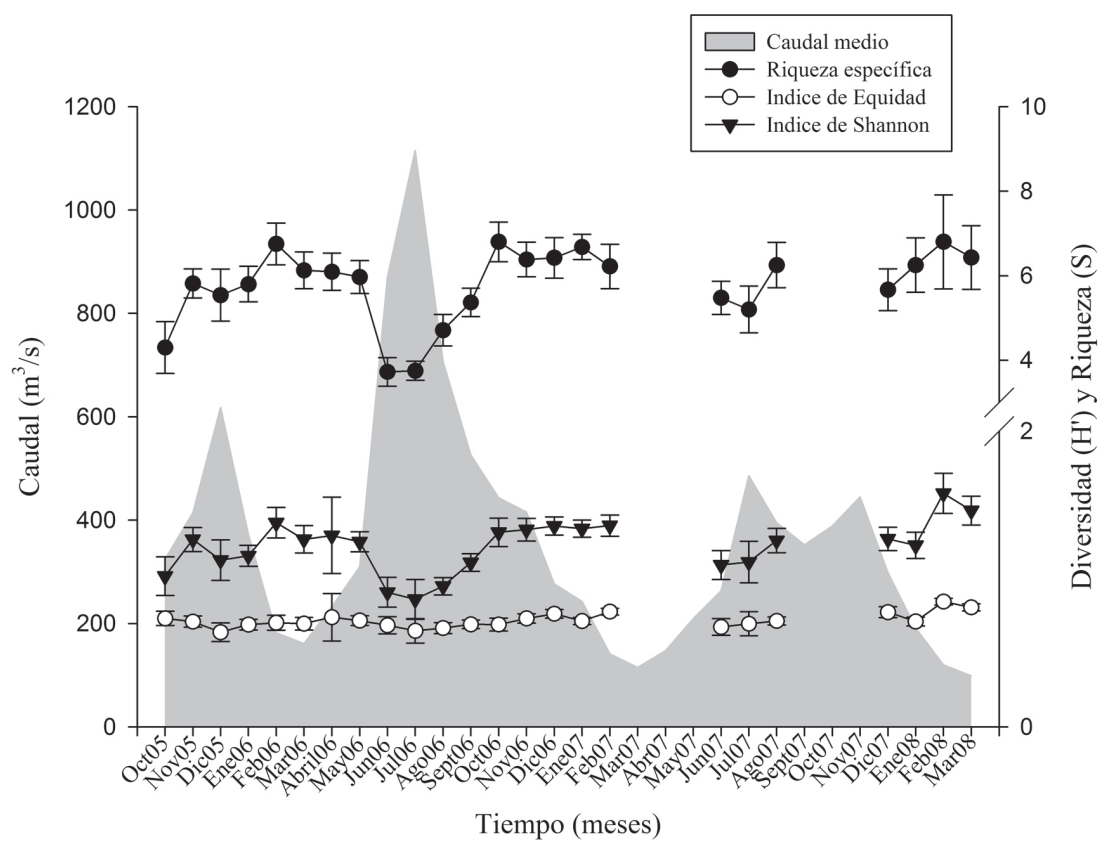

Figura 5. Tendencia de la riqueza de especies, diversidad específica (índices de Equidad y Shannon) y caudal medio mensual asociado desde octubre de 2005 a marzo de 2008. Los meses sin datos corresponden a ausencia de muestreos $(\mathrm{N}=31344)$.

FIGURE 5. Species richness, diversity (Evenness y Shannon indexes) and monthly average flow trends from Octuber 2005 to March 2008. Months without values did not have samples $(\mathrm{N}=31344)$.

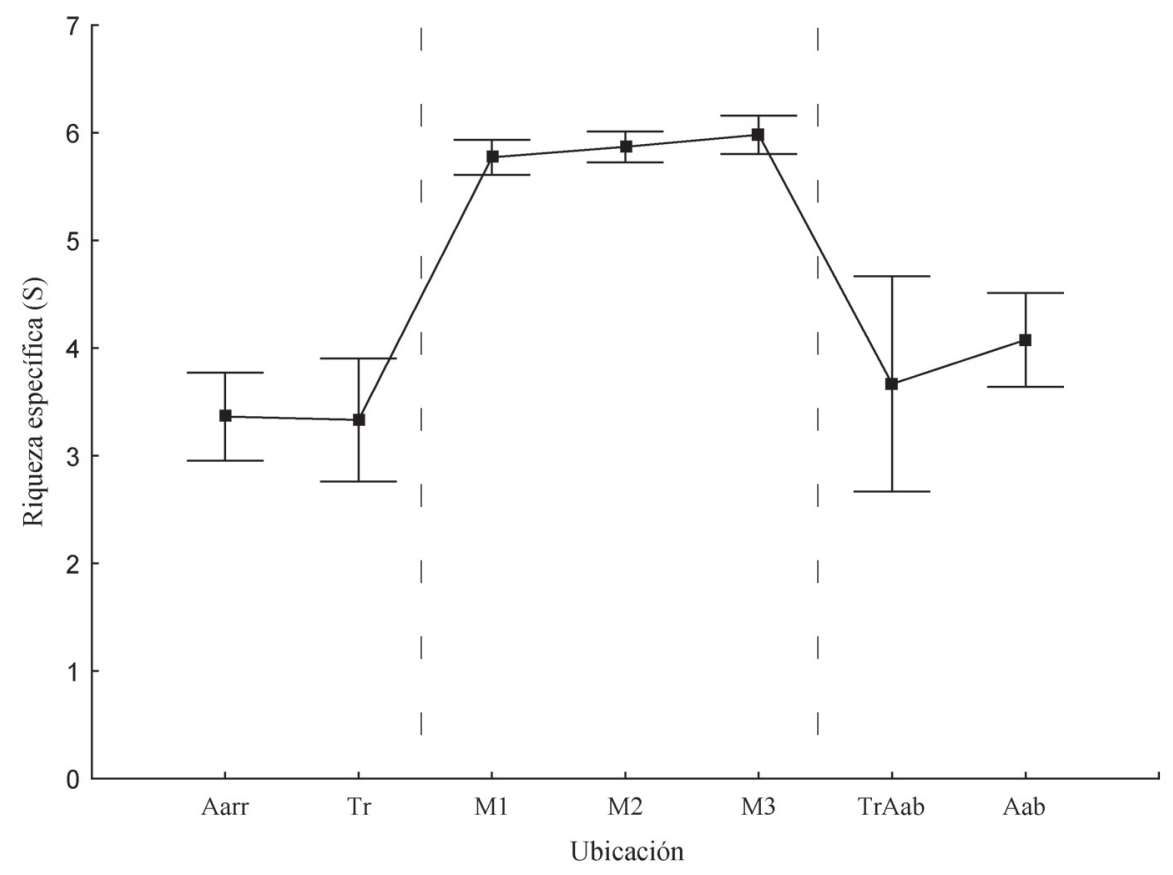

Figura 6. Riqueza de especies en las distintas zonas muestreadas de la cuenca del río Valdivia. Las líneas punteadas indican el tramo correspondiente al río San Pedro. Aarr: ríos aguas arriba del San Pedro, Tr: tributarios del San Pedro, M1 - M3: macrozonas 1 a la 3 del río San Pedro, TrAab: tributarios aguas abajo del San Pedro, Aab: río aguas abajo del San Pedro (desde confluencia con el río Quinchilcas).

FIgURA 6. Species richness in different zones sampled of the Valdivia river basin. Dotted lines show the San Pedro river length. Aarr: upstream, Tr: tributaries of the San Pedro River, M1 - M3: macrozones 1 to 3 of the San Pedro River, TrAab: dowstream tributaries of San Pedro River, Aab: The San Pedro River dowstream (from confluence with Quinchilca River). 
Patrones comunitarios de peces del río San Pedro: Nicole Colin ET AL.

TABLA 2. Abundancia relativa promedio de cada especie por macrozona y porcentaje de contribución a la similitud de las macrozonas (entre paréntesis). Se muestra también la disimilitud promedio entre macrozonas y el porcentaje de contribución de las especies más abundantes. En negrita las especies con mayor contribución.

TABLE 2. Average relative abundance of each species by macrozone and percentage of contribution to the macrozones similarity (in parenthesis). Average dissimilarity among macrozones and species contribution to the dissimilarity. In bold species with higher contribution.

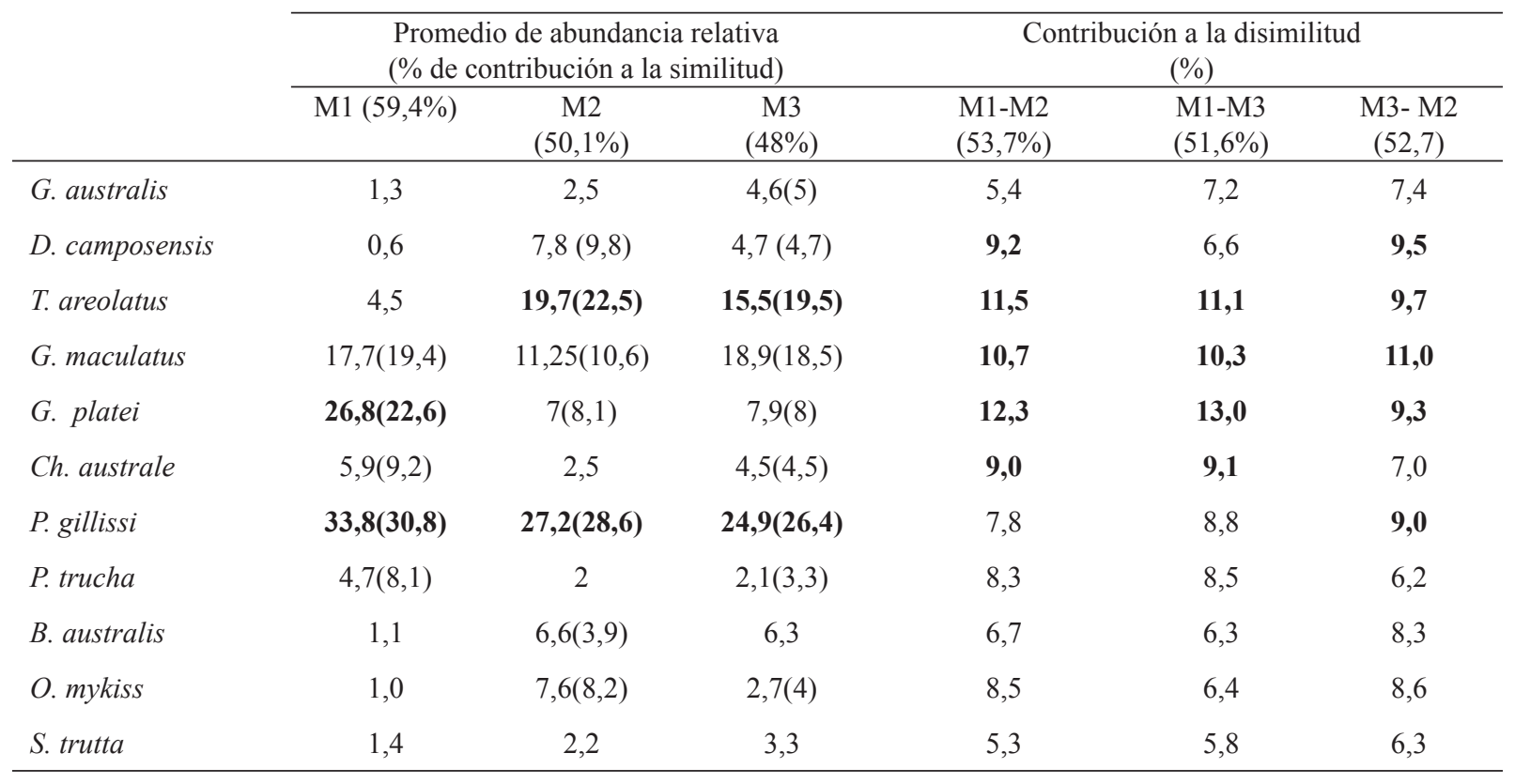

TABLA 3. Abundancia relativa promedio de cada especie por época y porcentaje de contribución a la similitud de las épocas (entre paréntesis). Se muestra también la disimilitud promedio entre épocas y la contribución a ella de cada especie. En negrita las especies con mayor contribución. AQ: época de alto caudal, BQ: época de bajo caudal.

TABLE 3. Average relative abundance of each species by season and percentage of contribution to the season similarity (in parenthesis). Average dissimilarity between seasons and species contribution to the dissimilarity. In bold species with higher contribution. AQ: high flow season, BQ: low flow season.

\begin{tabular}{|c|c|c|c|}
\hline & \multicolumn{2}{|c|}{$\begin{array}{l}\text { Promedio de abundancia relativa } \\
\text { (\% de contribución) }\end{array}$} & \multirow{2}{*}{$\begin{array}{c}\text { Porcentaje a la disimilitud } \\
(\%) \\
\text { AQ-BQ }\end{array}$} \\
\hline & AQ & BQ & \\
\hline G. australis & 1,9 & $4(3,7)$ & 6,8 \\
\hline D. camposensis & $3,5(3,4)$ & $8,3(9)$ & 9,4 \\
\hline T. areolatus & $11,3(14,2)$ & $21,4(23,7)$ & 10,5 \\
\hline G. maculatus & $21,1(22,5)$ & $7,5(8,2)$ & 11,2 \\
\hline G. platei & $13,9(13,1)$ & $21,4(7,4)$ & 10,2 \\
\hline Ch. australe & $3,7(3,3)$ & 3,4 & 7,1 \\
\hline P. gillissi & $27,7(29,6)$ & $27,2(28,3)$ & 8,6 \\
\hline P.trucha & 1,9 & 4,0 & 6,7 \\
\hline B. australis & $3,1(5,2)$ & 8,3 & 8,3 \\
\hline O. mykiss & $4,6(5)$ & $5,9(6,5)$ & 8,5 \\
\hline S. trutta & 3,2 & 1,7 & 6,0 \\
\hline
\end{tabular}


Comparativamente con el río San Pedro todos los sitios muestreados en otros sectores de la cuenca (aguas arriba, aguas abajo y tributarios), mostraron menor número de especies nativas, siendo los tributarios del San Pedro los que poseen menor riqueza de especies (Fig. 6). Las comunidades del río San Pedro (macrozonas 1, 2 y 3), se comportaron como una unidad homogénea al compararlas con aquellas presentes fuera del río (Fig. 7). Los ríos aguas arriba presentan una baja riqueza, predominando especies como salmónidos y A. zebra. En general, las diferencias entre las comunidades de peces presentes aguas arriba del río San Pedro y aquellas que habitan en el río San Pedro (70,3\% de disimilitud, basado en SIMPER) se explican principalmente por la mayor abundancia de $P$. gillissi, $T$. areolatus, $G$. maculatus, G. platei y D. camposensis en el río San Pedro versus los sitios aguas arriba donde éstos son menos abundantes. Aguas abajo (Aab) existe una disminución de la abundancia de especies principalmente ritrónicas $(57,7 \%$ de disimilitud), como P. gillissi, T. areolatus, D. camposensis y Oncorhynchus mykiss Walbaum, 1792.

Por último, las comunidades ícticas del río San Pedro presentan grandes diferencias con aquellas comunidades de sus tributarios ( $\mathrm{Tr})$ (72,9\% de disimilitud). En primer lugar, estas diferencias se explican por la ausencia en los tributarios de las especies nativas Aplochiton taeniatus Jenyns, 1842, A. zebra, Basilichthys australis Eigenmann, 1927, Ch. australe, Odontesthes mauleanum Steindachner, 1896 y Percichthys trucha Valenciennes, 1833, así como por la mayor abundancia de $P$. gillissi en el río San Pedro y de los salmonídeos en los tributarios.

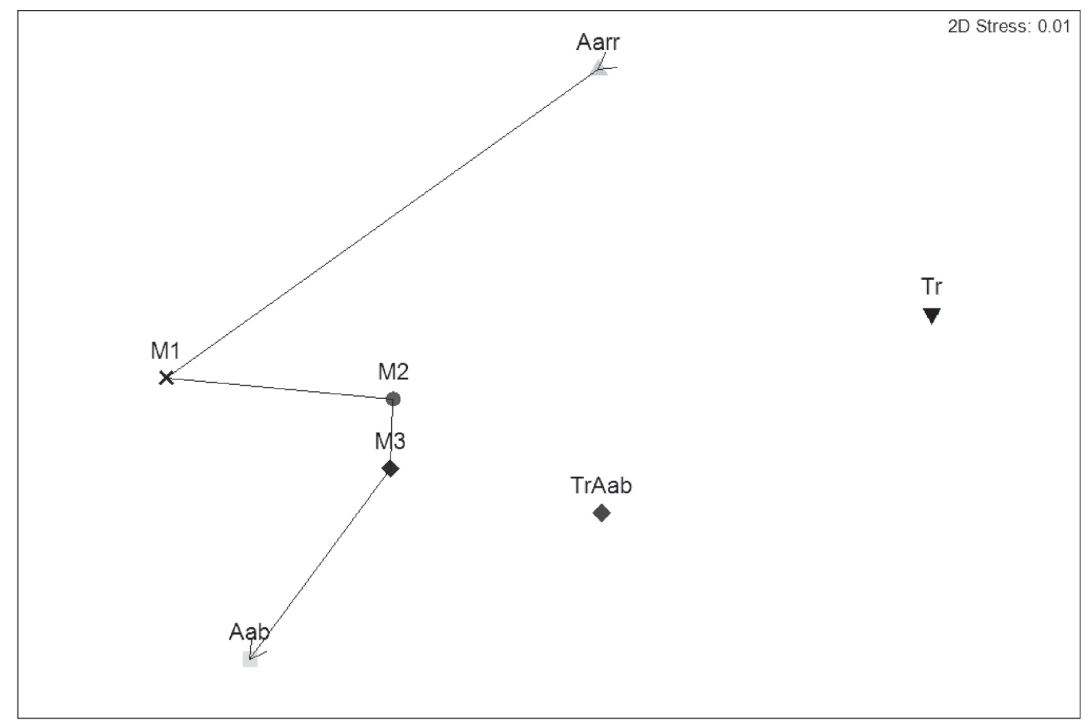

FiguRA 7. Análisis de escalamiento multidimensional no métrico (NMDS) basado en la abundancia relativa y el índice de similitud de BrayCurtis. La trayectoria muestra la tendencia unidireccional de las comunidades de peces desde los sectores ubicados aguas arriba del San Pedro (Aarr), río San Pedro en macrozona 1 (M1), macrozona 2 (M2), macrozona 3 (M3) y ríos ubicados aguas abajo (Aab).

Figure 7. Multi-dimensional scaling analysis no metric (NMDS) based on relative abundance, and the Bray-Curtis similarity index. The trajectory shows the unidirectional tendency of the fish communities from the zones located dowstream (Aarr), San Pedro river in macrozone 1 (M1), macrozone 2 (M2), macrozone 3 (M3) and rivers located in dowstream (Aab).

\section{DISCUSIÓN}

La presencia del Lago Riñihue (y de la cadena de lagos Araucanos sensu Thomasson 1963), en el origen del río San Pedro tiene una marcada influencia en las características físicas y biológicas del sistema fluvial. Aún cuando el tramo principal es de características ritrales, el primer tramo posee un claro efecto lacustre por lo cual la ictiofauna muestra características similares a comunidades presentes en zonas potamales de ríos andinos (Habit et al. 2006). De esta forma, este río de corta extensión (40 km) alcanza una riqueza específica de peces mucho mayor a la descrita para los tramos ritrales de los principales ríos andinos de Chile (Habit et al. 2006). Consecuentemente, el patrón detectado con frecuencia en otros ríos, que consiste en el aumento gradual de la riqueza específica desde el origen hacia aguas abajo (Vannote et al. 1980; Habit et al. 2003; Parra et al. 2004; Lampert \& Sommer 2007), no se cumple. Estas características presentes solo en este sistema dentro de la cuenca, hacen de él un ecosistema con una alta singularidad, 
con una ictiofauna distinta a la del resto de la cuenca hidrográfica.

Dentro del río San Pedro, cada macrozona posee una estructuración comunitaria de la fauna de peces, con variaciones estacionales producto de las diferencias en el caudal. Ello se traduce en un alta dinámica en los ambientes ribereños de alto significado ecológico (Strayer \& Findlay 2010). De esta forma, las comunidades de peces presentes en el río San Pedro presentan una alta dinámica espacial y temporal, debido a los cambios en las condiciones ambientales tanto a lo largo del río, como a través del año. Estos factores actúan como filtros ambientales, los cuales permiten la existencia y abundancia de especies que poseen características adecuadas para cada tipo de ambiente (Keddy 1992; Poff 1997; Townsend et al. 1997; Belyea \& Lancaster 1999; Spooner \& Vaughn 2008; Hopkins \& Burr 2009). La variación de la pendiente en el sistema influye directamente en la magnitud de la velocidad de corriente, parámetro determinante en la morfología del hábitat debido a que influye en la variación de otras variables como el oxígeno, arrastre de material y sedimentación (sustrato) (Poff et al. 1997). De esta forma, la zona 1 y 3 están caracterizadas por velocidades menores, presencia de piedras y mayor sedimentación, en cambio la zona intermedia (2), posee velocidades más altas, mayor oxigenación del agua y presencia de bolones como sustrato. Ello se refleja en la conformación de comunidades ícticas distintivas a lo largo del río San Pedro, debido a la heterogeneidad y complejidad presente, la cual es consistente con las condiciones hidrogeomorfológicas del cauce (macrozonas). Esta heterogeneidad permite la existencia de una gran riqueza de especies, lo cual lo diferencia notablemente de los demás tramos de la cuenca. En el río San Pedro ocurren peces especialistas de diferentes tipos de ambiente, como por ejemplo $D$. camposensis, que habita principalmente el tramo de rápidos (Habit et al. 2009), por lo cual caracteriza la comunidad de la macrozona 2 . Situación contraria sucede con $P$. gillissi, especie generalista en el uso de hábitats (Habit 1998), que por su alta abundancia caracteriza todas las zonas del río San Pedro.

Así como existe una estructuración comunitaria de la ictiofauna a nivel espacial (a lo largo del río), también existe a nivel temporal, con un claro patrón cíclico que genera una dinámica temporal marcada a lo largo del año. De esta forma, la variabilidad del régimen natural de caudal a través del tiempo, caracterizada principalmente por cinco componentes (magnitud, frecuencia, duración, previsibilidad y rango de cambio), es el factor fundamental para la presencia de una gran diversidad de hábitats y por ende de especies en el ecosistema fluvial (Poff et al. 1997). De acuerdo a los tipos de sistemas fluviales descritos por Poff \& Ward (1989), utilizando los componentes mencionados arriba, el río San Pedro esta dentro del grupo de los ríos con influencia pluvionival, el cual es caracterizado por una mayor frecuencia de inundaciones, una menor previsibilidad de éstas y de la magnitud del caudal, y por último, una mayor duración de las planicies de inundación. Por otra parte, la descripción de las funciones ecológicas asociadas a estos componentes pone mayor énfasis a los eventos de alto y bajo caudal, en este caso relacionados directamente con la presencia o ausencia de planicies de inundación, debido a que estos usualmente sirven como cuellos de botella ecológicos dado que presentan estreses críticos y oportunidades para un amplio orden de especies ribereñas (Poff \& Ward 1989; Poff et al. 1997). En este contexto, la riqueza y diversidad de especies son mayores en la época de bajo caudal cuando existe una mayor conexión entre los ensambles del canal central y la ribera, debido a la menor amplitud del cauce y disminución de la profundidad. En contraste, en la época de alto caudal, existe un cambio en la composición de los habitas ribereños, debido al aumento de la profundidad en el canal central y la inundación de ambientes terrestres (Strayer \& Frindlay 2010). Ello trae consigo un aumento de la amplitud del lecho (Sullivan \& Watzin 2009), incrementando la dispersión de las comunidades (Li \& Gelwick 2005; SaintPaul et al. 2000). En esta época de alto caudal, las diferencias físicas entre las planicies de inundación y el canal central, influyen en la distribución y abundancia de los peces en los ambientes ribereños (Tockner et al. 2010). Algunas especies, como los siluriformes, permanecen en el fondo del canal central del río, también como respuesta al decrecimiento de la temperatura superficial (Moring \& Nicholson 1994). En cambio otras especies utilizan las planicies de inundación que se generan en los pulsos de alto caudal (Scholosser 1991; Amsler et al. 2007; Lampert \& Sommer 2007; Rossi et al. 2007; Zeug \& Winemiller 2007a, 2007b, 2008). Este es el caso de G. maculatus, que se reproduce en tales ambientes en la época de alto caudal (Mitchell 1991; Hickford et al. 2010). De esta forma, los cambios estacionales implican una redistribución de las poblaciones de peces, existiendo comunidades que caracterizan las épocas de alto y bajo caudal, lo cual está asociado directamente con su presencia o ausencia en ambientes inundados (Scholosser 1991; Jowett \& Richardson 1994).

En este sentido, la dinámica comunitaria de la ictiofauna del río San Pedro, es la esperable de sistemas fluviales de régimen pluvionival no alterados (Poff \& Ward 1989; Poff et al. 1997; Sullivan \& Watzin 2009), donde el uso de las planicies de inundación por parte de los peces es de gran relevancia ecológica (Faush et al. 2002). Así por ejemplo, las planicies inundadas aumentan la disponibilidad de alimento dada la presencia de organismos terrestres que son consumidos por los peces (Scholosser 1991; Agostinho \& Zalewski 1995; Balcombe et al. 2005; Zeug \& Winemiller 2008). Además, es un hábitat importante para la reproducción 
de algunas especies (Copp \& Penáz 1988; Copp 1989; Jurajda 2006; Dibble \& Pelicice 2010), especialmente para aquellas que utilizan la vegetación terrestre para la postura de huevos (Dibble \& Pelicice 2010), como G. maculatus (Pollard 1971; Mitchell 1991; Hickford et al. 2010) y B. australis (Montoya et al. 2011). La evidencia generada aquí corrobora la importancia de la interacción del ecosistema terrestre con el acuático para la mantención de la dinámica y procesos ecológicos de las comunidades de peces y biota acuática en general (Copp \& Penáz 1988; Copp 1989; Schlosser 1991; Zeug \& Winemiller 2007a, 2007b, 2008).

En conclusión, las características hidrogeomorfológicas como pendiente (y velocidad), concentración de oxígeno disuelto y tipo sustrato principalmente, de cada macrozona evaluada, influyen en la distribución de las especies presentes, conformándose ensambles particulares para cada una de ellas. A nivel temporal, también existe un patrón de distribución de especies por los cambios en el caudal a lo largo del año, existiendo una comunidad característica para la época de alto caudal, caracterizada por presentar planos de inundación, así como para la de bajo caudal con ictiofauna característica del canal central. Por otra parte, al considerar al río San Pedro como una unidad ecológica, esta presenta una diversidad de especies notablemente mayor y una composición diferente en relación con los otros tramos de la cuenca, debido tanto a la heterogeneidad de hábitats presente en su cauce, como también a la influencia lacustre en el primer tramo del río (macrozona 1). Si a esto se suma la presencia mínima de especies introducidas (sólo 5\%), lo cual es poco frecuente en estos sistemas, es posible sugerir que el ecosistema presente en el río San Pedro posee una alta singularidad.

\section{AGRADECIMIENTOS}

Agradecemos a COLBUN S.A. por el financiamiento de este estudio y al proyecto DIUC Semilla Patagonia 210.310.0571SP por el financiamiento de la publicación.. A Catalina Puga del Hotel Riñimapu y propietarios de predios aledaños al río San Pedro, por su colaboración en la realización del trabajo de campo. Finalmente agradecemos al proyecto Fondecyt 1110441 que permitió finalizar el manuscrito.

\section{BIBLIOGRAFÍA}

Agostinho, A. \& Zalewski, M. 1995. The dependence of fish community structure and dynamics on floodplain and riparian ecotone zone in Parana River, Brazil. Hydrobiologia 303: 141-148.

Amsler, M., Drago, E. \& Paira, A. 2007. Fluvial Sediments: Main Channel and Floodplain Interrelationships. En: The Middle Paraná River: Limnology of a Subtropical Wetland. Eds. Iriondo. M., Paggi J.C. \& Parma, M., Springer-Verlag,
Berlin. pp. 363-377.

Balcombe, S., Bunn, S., Mckenzie-Smith, F. \& Davies, P. 2005. Variability of fish diets between dry and flood periods in an arid zone floodplain river. Journal of Fish Biology 67: $1552-1567$.

Belyea, L.R. \& Lancaster, J. 1999. Assembly Rules within a CONTINGENT ECOLOGY. OIKOS 86: 402-416.

Campos, H., Ruiz, V.H., Gavilán, J.F. \& Alay, F. 1993. Peces del río Biobío. Serie Publicaciones de Divulgación EULA, Editorial Universidad de Concepción, Concepción, Chile. $100 \mathrm{pp}$

Clarke, K. \& Gorley, R. 2006. PRIMER v6: User Manual/ Tutorial. PRIMER-E, Plymouth UK.

Copp, G. \& PenÁz, M. 1988. Ecology of fish spawning and nursery zones in the flood plain, using a new sampling approach. Hydrobiologia 169:209-224.

Copp, G. 1989. The habitat diversity and fish reproductive function of floodplain ecosystems. Environmental Biology of Fishes 26: 1-27.

Dibble, F. \& Pelicice, E. 2010. Influence of aquatic plant-specific habitat on an assemblage of small neotropical floodplain fishes. Ecology of Freshwater Fish 19: 381-389.

DYER, B. 2000. Systematic review and biogeography of the freshwater fishes of Chile. Estudios Oceanólogicos 19: 77 -98 .

Fausch, K., Torgersen, C., Baxter, C. \& Li, H. 2002. Landscapes to riverscapes: bridging the gap between research and conservation of stream fishes. BioScience 52(6): 483-498.

García de Jalón, D. \& GonzÁlez, M. 1998. Restauración de ríos y riberas. Ediciones Mundi-Prensa, Madrid, España. 317 pp.

Habit E. 1998. Análisis de la dieta de Percilia gillissi (Pisces: Perciliidae) en poblaciones de río y canales de riego (Cuenca del Itata, VIII Región). Theoria 7: 33-46.

Habit, E., Victoriano, P. \& Rodriguez-Ruiz, A. 2003. Variaciones espacio-temporales del ensamble de peces de un sistema fluvial de bajo orden del centro-sur de Chile. Revista Chilena de Historia Natural 76: 3-14.

Habit, E., Belk, M., Tuckfield, R. \& Parra, O. 2006. Response of the fish community to human-induced changes in the Biobío River in Chile. Freshwater Biology 51(1): 1-11.

Habit, E., Belk, M. \& PARRA, O. 2007. Response of the riverine fish community to the construction and operation of a diversion hydropower plant in Central Chile. Aquatic Conservation: Marine and Freshwater Ecosystems 17(1): 37-49.

Habit, E., Jara, A., Colin N., Oyanedel, A., Victoriano, P. GonzÁlez, J. \& Solis-Lufí, K. 2009. Threatened fishes of the World: Diplomystes camposensis Arratia, 1987 (Diplomystidae). Environmental Biology of Fishes 84(4): 393-394.

Hickford, M., Cagnon, M. \& Schiel, D. 2010. Predation, vegetation and habitat-specific survival of terrestrial eggs of a diadromous fish, Galaxias maculatus (Jenyns, 1842). Journal of Experimental Marine Biology and Ecology 385(1-2): 66-72.

Hopkins, R. \& BurR, B. 2009. Modeling freshwater fish distributions using multiscale landscape data: A case study of six narrow range endemics. Ecological Modelling 220: 2024-2034.

Jowett, I \& Richardson, J. 1994. Comparison of habitat use by fish in normal and flooded river conditions. New Zealand 
Journal of Marine and Freshwater Research 28(4): 409 $-416$.

Junk, W., Bayley, P. \& Sparks, R. 1989. The flood pulse concept in river-floodplain systems. Fisheries and Aquatic Sciences 106: 110-127.

JuRAJDA, P. 2006. Effect of channelization and regulation on fish recruitment in a flood plain river. Regulated Rivers Research and Management 10(2-4): 207-215.

Keddy, P. 1992. Assembly and Response Rules: Two Goals for Predictive Community Ecology. Journal of Vegetation Science 3(2): 157-164.

LAMPERT, W. \& SOMmer, U. 2007. Limnoecology: the ecology of lake and stream. Second edition, Oxford University press, Oxford.324 pp.

LI, R. \& Gelwick, F. 2005. The relationship of environmental factors to spatial and temporal variation of fish assemblages in a floodplain river in Texas, USA. Ecology of Freshwater Fish 2005: 14: 319-330.

MADDOK, I. 1999. The importance of physical habitat assessment for evaluating river health. Freshwater Biology 2: 373391.

Mitchell, C. 1991. Deposition of Galaxias fasciatus eggs with Galaxias maculatus eggs at a tidal site. New Zealand Journal of Marine and Freshwater Research 25: 201-205.

Montoya, G., Jara, A., Solis-Lufí, K., Colin, N. \& Habit, E. 2011. Descripción de huevos y larvas de peces nativos del río san pedro (Cuenca del río Valdivia, Chile). Gayana (en prensa).

Moring, J. \& Nicholson, P. 1994. Evaluation of three types of artificial habitats for fishes in a freshwater pond in Maine, USA. Bulletin of Marine Science 55(2-3): 1149-1159.

Newson, M. \& Newson, C. 2000. Geomorphology, ecology and river channel habitat: mesoscale approaches to basin-scale challenges. Progress in Physical Geography 24(2): 195217.

Niemeyer, H. \& P. Cereceda. 1984. Hidrografía. Geografía de Chile. Tomo VIII. Instituto Geográfico Militar. Chile.

Parra, O., Della Croce, N. \& Valdovinos, C. 2004. Elementos de limnología teórica y aplicada. Microart's Edizioni, Italia. 303 pp.

Poff, N. \& WARD, J. 1989. Implications of streamflow variability and predictability for lotic community structure: a regional analysis of streamflow patterns. Canadian Journal of Fisheries and Aquatic Sciences 46(10): 1805-1818.

Poff, N. 1997. Landscape filters and species traits: towards mechanistic understanding and prediction in stream ecology. Journal of the North American Benthological Society 16(2): 391-409.

Poff, N., Allan, J., Bain, M., Karr J., Prestegaard, K., Richter, B., Sparks, R. \& Stromberg, J. 1997. The natural flow regime: a paradigm for river conservation and restoration. BioScience 47: 769-784.

Pollard, D. 1971. The biology of a landlocked form of the normally catadromous salmoniform fish Galaxias maculatus (Jenyns): Life cycle and origin. Australian Journal of
Marine and Freshwater Research 22(2): 91-124.

Rossi, L., Cordiviola, E. \& Parma, M. 2007. Fishes. En: The Middle Paraná River: Limnology of a Subtropical Wetland (Eds. Iriondo, M., Paggi, J.C. \& Parma, M.), SpringerVerlag, Berlin. 363-377 pp.

Saint-Paul, U., Zuanon, J., Correa, M., Garcia, M., Fabre, N., Berger, U. \& JunK, W. 2000. Fish communities in central Amazonian white- and blackwater floodplains. Environmental Biology of Fishes 57: 235-250.

SCHLOSSER, I.J. 1991. Stream fish ecology: a landscape perspective. BioScience 41: 704-712.

Spooner, D. \& Vaughn, C. 2008. Trait-based approach to species' roles in stream ecosystems: climate change, community structure, and material cycling. Oecologia 158: 307-317.

Strayer, D. \& Findlay, S. 2010. Ecology of freshwater shore zones. Aquatic Science 72: 127-163.

Sullivan, S. \& Watzin, M. 2009. Stream-floodplain connectivity and fish assemblage diversity in the Champlain Valley, Vermont, U.S.A. Journal of Fish Biology 74: 1394-1418.

Thomasson, K. 1963. Araucanian lakes. Plankton studies in North Patagonia with notes on terrestrial vegetation. Acta Phytogeographica Suecica 47: 1-139.

Thorp, J., Thoms, M. \& Delong, M. 2006. The riverine ecosystem synthesis: biocomplexity in river networks across space and time. River Research and Applications 22: 123-147.

TOCKNER, K., LorAng, M. \& StANDFORD, J. 2010. River flood plains are model ecosystems to test general hydrogeomorphic and ecological concepts. River Research and Applications 26: 76-86.

Townsend, C., Dolédec, S. \& Scarsbrook, M. 1997. Species traits in relation to temporal and spatial heterogeneity in streams: a test of habitat templet theory. Freshwater Biology 37(2): 367-387.

Vannote, R., Minshall, G., Cummins, K., Sedell, J. \& Cushing, C. 1980. The river continuum concept. Canadian Journal of Fisheries and Aquatic Sciences 37: 130-137.

Vila, I., PArdo, R., Dyer, B. \& Habit, E. 2006. Peces límnicos: diversidad, origen y estado de conservación. En: Macrófitas y vertebrados de los sistemas límnicos de Chile (Eds. Irma, V., Veloso, A., Shlatter, R. \& Ramírez, C.), pp. 73-102. Editorial Universitaria, Santiago, Chile.

WARD, J. 1989. The four-dimensional nature of lotic ecosystems. Journal of the North American Benthological Society 8(1): 2-8.

Zeug, S \& Winemiller, K. 2007(a). Ecological correlates of fish reproductive activity in floodplain rivers: a life-historybased approach. Canadian Journal of Fisheries and Aquatic Sciencies 64(10): 1291-1301.

Zeug, S. \& Winemiller, K. 2007(b). Relationships between hydrology, spatial heterogeneity, and fish recruitment dynamics in a temperate floodplain river. River Research and Application 24(1): 90-102.

Zeug, S. \& Winemiller, K. 2008. Evidence supporting the importance of terrestrial carbon in a large-river food web. Ecology 89: 1733-1743.

Recibido: 02.01.12

Aceptado: 27.02 .12 